\title{
Optically Induced Damping of the Surface Plasmon Resonance in Gold Colloids
}

\author{
M. Perner, P. Bost, U. Lemmer, G. von Plessen, and J. Feldmann \\ Sektion Physik, Ludwig-Maximilians-Universität München, Amalienstrasse 54, D-80799 München, Germany
}

\author{
U. Becker, M. Mennig, M. Schmitt, and H. Schmidt \\ Institut für Neue Materialien, Im Stadtwald, D-66123 Saarbrücken, Germany
}

(Received 30 August 1996)

\begin{abstract}
The surface plasmon damping induced by high excitation of the electron gas is studied in femtosecond pump-and-probe experiments on gold colloids embedded in a sol-gel matrix. Optical excitation of single-particle interband transitions leads to a pronounced broadening of the surface plasmon line. A similar behavior is observed for resonant excitation of the surface plasmon. This broadening is the dominant optical nonlinearity of the system, and reflects the excitation-induced damping of the surface plasmon resonance. The time evolution of the damping rate follows that of the electronic scattering rate. [S0031-9007(97)02694-X]
\end{abstract}

Surface plasmons (SPs) are collective electronic excitations at the interface between a metal and a dielectric. They play an important role in metal films [1], in semiconductor heterostructures [2], and in metal nanoparticles [3]. SPs show pronounced optical absorption resonances due to coupling of the light to the collective electronic excitation. A large number of applications in decorative coloring [3], in the biochemical field [4], and in optical devices [5] are based on SP resonances.

The properties of the SP are crucially influenced by its lifetime. Decay, or damping, mechanisms of the SP have been extensively studied in the literature. Quantum mechanically, the decay involves the interaction of the SP with single-particle excitations [1]. In a semiclassical picture, the decay is often described in terms of a loss of phase coherence of the collective electron oscillation due to scattering of single electrons [3]. This theoretical understanding is consistent with optical spectra of metal colloids that show a strong correlation between the SP linewidth and the calculated rate of electronic surface scattering [3], and with recent timeresolved optical experiments on Ag films [6,7]. All experimental studies of the SP decay in metals have so far been performed in the low-excitation regime, where the energy optically transferred to the system is too small to strongly perturb the single-particle distribution. In contrast, no clear information is available on the SP decay in metals under high-excitation, nonequilibrium conditions. This physical situation is of high interest, both for fundamental reasons and with a view to device applications where large energies are optically transferred to the system.

In this Letter, we study the damping of the SP in gold nanoparticles induced by high excitation of the electron gas. We perform femtosecond pump-and-probe experiments on gold colloids embedded in a dielectric sol-gel matrix. The excitation of the electron gas is provided by optical pumping of single-particle interband transitions from the $d$ band into $s$ - $p$ states far above the Fermi level. We observe a SP line broadening which builds up within $\sim 1$ ps after excitation. A similar behavior is observed for resonant excitation of the SP. In both cases, the broadening is the absolutely dominant optical nonlinearity and reflects the excitationinduced damping of the SP resonance. The time evolution of the damping rate is found to essentially follow that of the total electronic scattering rate expected from comparison with other experiments and theoretical considerations.

Metal colloids show pronounced SP resonances, whose spectral position can be varied over a wide range by a suitable choice of the metal, the dielectric, and the geometry of the colloid [3]. For the present work, gold colloids have been prepared in a photocurable dielectric inorganic-organic nanocomposite matrix by solgel processing [8]. The samples studied here consist of a $2.6 \mu \mathrm{m}$ thick sol-gel composite film which contains spherical gold colloids with a mean diameter of $30 \mathrm{~nm}$ and a volume fill factor of $0.2 \%$. The optical density of this sample as a function of photon energy $E$ is shown in Fig. 1(a) (solid line). The resonance of the SP lies at $2.29 \mathrm{eV}$, below the interband (IB) absorption edge at $E_{d-E_{F}}=2.38 \mathrm{eV}$, and has a spectral width of $316 \mathrm{meV}$ [dotted line in Fig. 1(a)]. This width is controlled by homogeneous line broadening due to surface-scatteringinduced damping, and possibly also by inhomogeneous contributions due to fluctuations of the colloid size and local dielectric constant of the sol-gel matrix. The gradual increase of the optical density for higher energies [dashed line in Fig. 1(a)] is caused by single-particle IB transitions from the $5 d$ to the $6 s p$ band [9].

We use the frequency-doubled output of a regenerative Ti:sapphire amplifier ( $1 \mathrm{kHz}$ repetition rate) as the pump pulse and a white-light continuum as the probe pulse. 


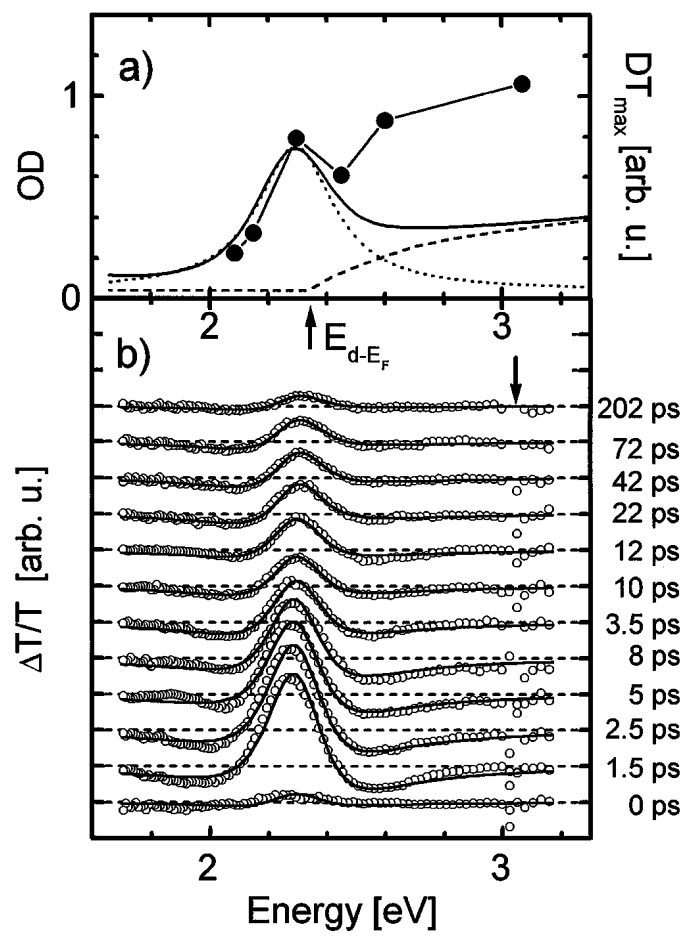

FIG. 1. (a) Optical density OD $=\log _{10} T$ vs photon energy (solid line). Dotted line: Lorentzian line shape fit of the SP resonance. Dashed line: single-particle interband absorption obtained from subtracting the dotted line from the solid line. Dots show maxima of induced transmission $D T_{\max }$ for different pump-photon energies. (b) Differential transmission $\Delta T / T$ versus probe-photon energy at different time delays $(\Delta T / T \approx \ln 10 \times \Delta \mathrm{OD})$. The arrow indicates the central photon energy of the pump pulse.

The pump beam has an average power of $2.4 \mathrm{~mW}$ and is focused down to a spot diameter of $300 \mu \mathrm{m}$; the pump pulse has a duration of $200 \mathrm{fs}$, and is centered at $3.07 \mathrm{eV}$, as indicated by the arrow in Fig. 1(b). Alternatively, pump pulses with a duration of $<200 \mathrm{fs}$ and central photon energies between 2.08 and $2.6 \mathrm{eV}$ were generated by a tunable optical parametric amplifier. The white-light continuum is generated by focusing part of the laser power into a water flow cell, and has a temporal duration of 200 fs. The probe pulse can be delayed with respect to the pump pulse by a variable time delay $\tau$, and is spectrally analyzed in a monochromator after transmission through the sample.

Differential transmission (DT) spectra $\Delta T / T(h \omega, \tau)$ were taken at a sample temperature of $35 \mathrm{~K}$, and for optical excitation at a photon energy of $3.07 \mathrm{eV}$, i.e., in the spectral region of IB transitions. A set of such spectra taken for a series of time delays $\tau$ is plotted in Fig. 1(b) versus the energy of probe photons $h \omega$. We observe a pronounced nonlinearity at the spectral position of the SP, which reaches peak values of up to $\Delta T / T \approx 15 \%$. This nonlinearity consists of regions of induced transmission (center of the SP line) and induced absorption (wings), respectively. It is mainly caused by spectral broadening of the resonance, as can be seen from the signal shape in Fig. 1(b). A similar behavior is observed upon direct excitation of the SP (not shown here). The DT spectra are fitted assuming for simplicity a purely Lorentzian line shape of the resonance [3]. This approach amounts to including in the Lorentzian line shape any linewidth contributions from inhomogeneous broadening effects; since we are interested only in the excitation-induced increase of the homogeneous linewidth, this approximation is expected to work reasonably well for all our present purposes. In the fit procedure, we use the linewidth $\Gamma$, the spectral position of the plasmon resonance, and the oscillator strength as adjustable parameters. The results of the line shape fit confirm that the dominant contribution to the measured nonlinearity originates from spectral broadening; minor contributions come from changes in the spectral position and oscillator strength [10].

The time dependence of the broadening for IB excitation at $3.07 \mathrm{eV}$ is shown in Fig. 2, where $\Gamma$ is plotted as a function of time delay. Within the first picosecond after excitation, we observe a rapid initial rise of the linewidth from the equilibrium value of $316 \mathrm{meV}$ to a maximum value of $432 \mathrm{meV}$. The subsequent return of $\Gamma$ to the equilibrium value is characterized by a twocomponent decay with time constants of 4 and $\sim 200 \mathrm{ps,}$ respectively. For an explanation of this behavior, we note that a similar sequence of rapid initial rise and subsequent two-component decay has been observed in the electron temperature in metal films after fs laser excitation [11]. Such temperature changes are accompanied by variations in the electronic scattering rate [12]. We suggest that the increased line broadening shown in Fig. 2 is a result of SP damping due to such variations in the electronic scattering rate. In more detail, our interpretation of the time dependence of the SP linewidth for IB excitation is as follows.

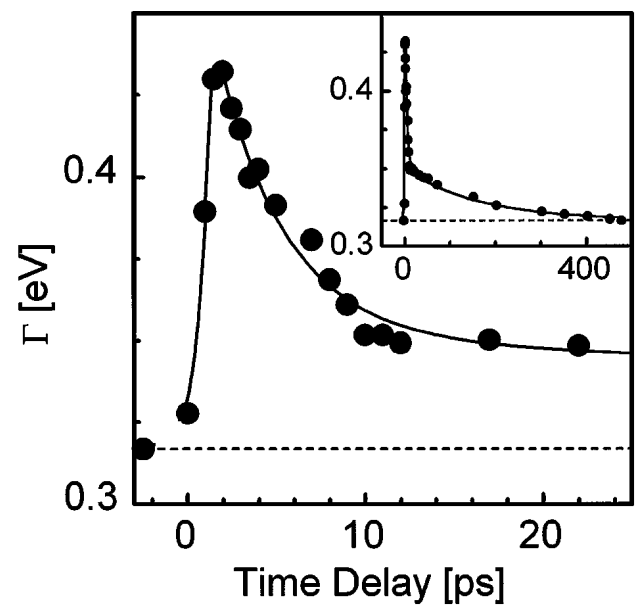

FIG. 2. Temporal evolution of the plasmon linewidth after interband excitation; dashed line: equilibrium value of the plasmon linewidth $(316 \mathrm{meV})$. Inset: The same plot on a larger time scale. 
(i) The pump pulse induces single-particle transitions to $6 s p$-band states far above the Fermi level, and thus creates a nonthermal distribution of optically excited electrons. The electron gas thermalizes within $\sim 1$ ps via electron-electron and electron-phonon scattering [11,1315]. During the thermalization process, the excess energy of the optically excited particles is redistributed among the conduction band electrons. The Fermi edge smearing associated with this heating process (solid line in Fig. 3) results in an enhanced probability for electron scattering between states in the vicinity of the Fermi energy, due to the high density of both populated initial and unpopulated final states. The enhancement of the total electronic scattering rate leads, via the interaction between the SP and the single-particle excitations, to an increase of the SP decay rate that manifests itself as a line broadening in the DT spectra in Fig. 1(b).

(ii) During and after thermalization, the highly excited electron gas loses energy to the gold lattice by phonon emission until the temperatures of the electron distribution and the lattice have equalized. Concomitant with this process is an increasing limitation of the electron scattering rate in accordance with the Pauli exclusion principle, due to the recovery of the energetic sharpness of the Fermi edge (dotted line in Fig. 3). The decay of the scattering rate leads to a decay of $\Gamma$ with a time constant of 4 ps; this value is typical of equilibration processes between electron and lattice temperatures in metal colloids [15-17].

(iii) The temperature equilibration between the electron gas and the gold lattice leaves the joint system at a higher temperature than before the pump pulse; this is reflected in a nonvanishing line broadening at time delays $\tau>10 \mathrm{ps}$. The gradual decay of $\Gamma$ with a time constant of $200 \mathrm{ps}$ is explained by heat transfer from the gold colloids to the embedding matrix $[16,17]$.

In steps (i)-(iii), the observed time evolution of the SP damping rate after IB excitation qualitatively follows that of the expected electronic scattering rate. In the following, we will attempt a quantitative comparison

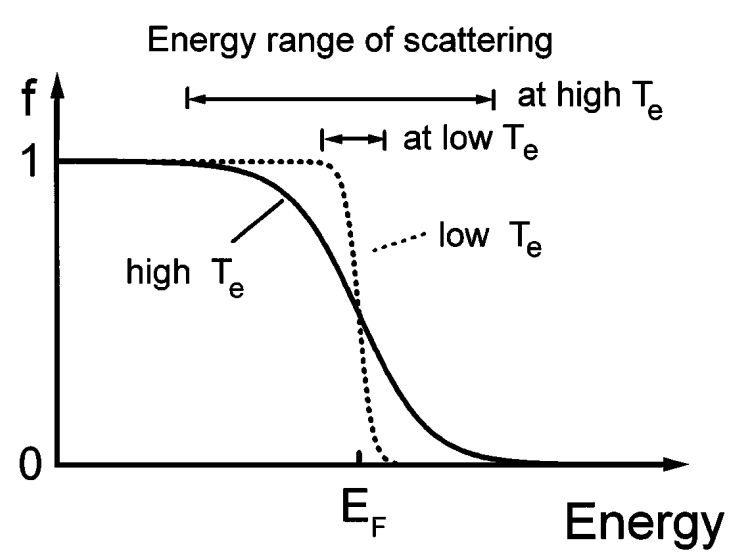

FIG. 3. Schematic Fermi distribution functions at low (dashed line) and high (solid line) electron temperatures. between the two rates immediately after thermalization, i.e., at the end of the step (i). The experimental linewidth $\Gamma$ in Fig. 2 is observed to increase by as much as $120 \mathrm{meV}$ during step (i), which corresponds to an increase of $(10 \mathrm{fs})^{-1}$ in the SP damping rate. According to Fermi liquid theory, the electron-electron $(e-e)$ scattering rate $\tau_{e e}^{-1}$ for an electron at excess energy $\varepsilon=E-E_{F}$ above the Fermi level is given by

$$
\frac{1}{\tau_{e e}}=K \frac{\left(\pi k_{B} T_{e}\right)^{2}+\varepsilon^{2}}{1+\exp \left(-\varepsilon / k_{B} T_{e}\right)}
$$

where $K=0.1 \mathrm{eV}^{-2} \mathrm{fs}^{-1}$ is the $e$-e scattering-rate constant according to Ref. [18], and $T_{e}$ is the temperature of the conduction band electrons. To gain some simple quantitative measure of the $e$-e scattering rate, we define an average $e$ - $e$ scattering rate as $\left\langle\tau_{e e}^{-1}\right\rangle=\int_{0}^{\infty} f(\varepsilon) \tau_{e e}^{-1}(\varepsilon) d \varepsilon / \int_{0}^{\infty} f(\varepsilon) d \varepsilon$, where $f(\varepsilon)=$ $1 /\left[1+\exp \left(\varepsilon / k_{B} T_{e}\right)\right]$ is the Fermi function. From the known experimental parameters, we calculate that each pump pulse excites $\sim 3 \times 10^{4}$ electrons per colloid from the $d$ bands into the $s p$ band, leaving behind an equal number of holes in the $d$ bands. For an estimate of $T_{e}$ at the end of step (i), it is important to know how fast the holes release their excess energy to the conduction band electrons and to the lattice by relaxing towards the Fermi level. Some answers to this problem can be obtained from the differential transmission at the spectral position of SP resonance, $\Delta T / T(h \omega=2.29 \mathrm{eV})$. This quantity is a direct measure of the excitation-induced increase in $\Gamma$, and thus of the SP damping; it reaches its maximum value, $D T_{\max }$, at the end of step (i). $D T_{\max }$ is plotted in Fig. 1(a) as dots versus pump photon energy. If the excess energy of the optically excited electrons with respect to the Fermi level was the only one to contribute to the electron heating, $T_{e}$ and hence the SP damping rate should be much higher for excitation at 3.07 than at $2.60 \mathrm{eV}$. As the corresponding values of $D T_{\max }$ show, this is not the case. We therefore conclude that a substantial part of the excess energy of the photogenerated holes is transferred to the conduction-band electrons (and the lattice) via fast scattering processes during the first ps after excitation. For simplicity we will assume in the present estimate that this actually applies to all the hole excess energy. We then obtain $T_{e} \approx 4000 \mathrm{~K}$ at the end of step (i), using the heat capacity of the electron gas as taken from Ref. [18]. From Eq. (1), we calculate an average $e$-e scattering rate of $\left\langle\tau_{e e}^{-1}\right\rangle=(10 \mathrm{fs})^{-1}$ for the hot electron distribution at $4000 \mathrm{~K}$, in very good agreement with the observed increase in the SP damping rate. This value certainly overestimates the true $e-e$ scattering rate somewhat since we have taken into account all the hole excess energy and neglected the heat transfer to the lattice in the estimate. However, even in view of these simplifications, the true value of the $e-e$ scattering rate will still be large enough to safely say that $e-e$ scattering can strongly influence the 
SP damping during thermalization of the highly excited electron gas. Conversely, the electron-phonon (e-ph) scattering rate is on the order of $\sim(0.1 \mathrm{ps})^{-1}$ [19], which implies that $e$-ph scattering probably gives a smaller contribution to the optically induced SP damping. The third electronic scattering process which can be strongly enhanced at high electron temperatures is electron-surface scattering, due to the high density of both populated initial and unpopulated final states at the same energy (Fig. 3). However, the influence of this scattering process on the damping rate under high-excitation conditions is at present difficult to quantify.

We finally note that a similar temporal dependence as in the case of IB excitation is observed for optical excitation at the spectral position of the SP (not shown here). This leads to the conclusion that also in this latter case, the SP damping is strongly influenced by electron scattering due to high excitation of the electron gas. However, an important difference is that here the electron gas is heated by the energy released when the SP excited by the pump pulse decays [18]. Another interesting difference becomes apparent when focusing on $D T_{\max }$ in Fig. 1(a). $D T_{\max }$ closely follows the measured optical density in the spectral region of the SP line. This is understandable since the total energy optically transferred to the nanoparticle is expected to be proportional to the number of absorbed photons. It is remarkable, however, that $D T_{\max }$ strongly deviates from the optical density when pumping takes place in the spectral range of the IB transitions. This means that pumping at interband transition frequencies results in a more efficient $S P$ damping than at the SP resonance. This difference can be explained when we assume that part of the energy stored in the SP mode by the resonant pump pulse is not directly released to the electron gas but is relaxed through different dissipative channels (e.g., via SP-phonon coupling).

Our explanation of the SP line broadening also casts a new light on observations made by other groups. In Refs. [15,17], transient broadenings of the SP line have been observed after detuned excitation of the SP resonance in pump-and-probe experiments on noble metal nanoparticles. While these studies include no quantitative analysis of the SP linewidth on which to base an unequivocal judgment, the published spectra hint towards a possible interpretation of the line broadenings in terms of an excitation-induced SP damping, in analogy to the case discussed in the present Letter.

In summary, we have performed the first study of surface plasmon decay induced by the interaction with a highly excited electron gas in a metallic system. A pronounced broadening of the surface plasmon line in gold colloids is observed both in the case of singleparticle interband excitation, and for direct excitation of the surface plasmon. This line broadening is by far the dominant optical nonlinearity of the system, and reflects the excitation-induced damping of the surface plasmon resonance. The transient increase of the damping rate is governed by the time evolution of the electronic scattering rate. We expect these results to be of importance for the understanding of the surface plasmon decay in all situations where large energies are optically transferred to the electron gas.

The experiments have been performed in the laboratories of E. O. Göbel at the University of Marburg. We thank M. Preis for expert technical assistance.

[1] D. Pines, Rev. Mod. Phys. 28, 184 (1956).

[2] E. L. Albuquerque and M. G. Cottam, Phys. Rep. 233, 67 (1993).

[3] U. Kreibig and M. Vollmer, Optical Properties of Metal Clusters (Springer-Verlag, Berlin, 1995).

[4] S. Lofas et al., Sens. Actuators B, Chem. 5, 79 (1991).

[5] J. W. Haus et al., J. Appl. Phys. 65, 1420 (1989).

[6] M. van Exter and A. Lagendijk, Phys. Rev. Lett. 60, 49 (1988).

[7] D. Steinmüller-Nethl et al., Phys. Rev. Lett. 68, 389 (1992).

[8] M. Mennig et al., in Proceedings of the 8th CIMTEC, Florence, 1994 (Techna, Faenza, 1995).

[9] R. Rosei and D. W. Lynch, Phys. Rev. B 5, 3883 (1972).

[10] For small time delays, the SP line is blueshifted by up to $\sim 20 \mathrm{meV}$. This blueshift decays with a time constant of $\sim 5 \mathrm{ps}$ and subsequently turns into a small redshift of $\sim 5 \mathrm{meV}$, which decays on a time scale of several hundred ps. The reasons for this transient line shift are at present not clearly known.

[11] C. K. Sun et al., Phys. Rev. B 50, 15337 (1994).

[12] D.-S. Kim et al., Phys. Rev. Lett. 68, 2838 (1992).

[13] W. S. Fann, R. Storz, and H. W. K. Tom, Phys. Rev. B 46, 13592 (1992).

[14] J. Y. Bigot et al., Phys. Rev. Lett. 75, 4702 (1995).

[15] T. S. Ahmadi, S. L. Logunov, and M. A. Elsayed, J. Phys. Chem. 100, 8053 (1996).

[16] T.W. Roberti, B. A. Smith, and J.Z. Zhang, J. Chem. Phys. 102, 3860 (1995).

[17] T. Tokizaki et al., Appl. Phys. Lett. 65, 941 (1994).

[18] R. H. M. Groeneveld, R. Sprik, and A. Lagendijk, Phys. Rev. B 51, 11433 (1995).

[19] C. Suárez, W. E. Bron, and T. Juhasz, Phys. Rev. Lett. 75, 4536 (1995). 